\title{
A Bond Bundle Case Study of Diels-Alder Catalysis Using Oriented Electric Fields
}

\author{
Timothy R. Wilson ${ }^{1,2}$ M.E. Eberhart ${ }^{1}$
}

December 20, 2021

\begin{abstract}
Bond bundles are chemical bonding regions, analogous to Bader atoms, uniquely defined according to the topology of the gradient bundle condensed charge density, itself obtained by a process of infinitesimal partitioning of the three-dimensional charge density into differential zero-flux surface bounded regions. Here we use bond bundle analysis to investigate the response of the charge density to an oriented electric field in general, and the catalytic effect of such a field on Diels-Alder reactions in particular, which in this case is found to catalyze by allowing the transition state valance bond bundle configuration to be achieved earlier along the reaction pathway. Using precise numerical values, we arrive at the conclusion that chemical reactions and electric field catalysis can be understood in terms of intra-atomic charge density redistribution, i.e., that charge shifts within more so than between atoms account for the making and breaking of bonds.
\end{abstract}

\section{Introduction-Atomic basins and bond bundles}

Bader's most significant observation was that regions of electron charge density $(\rho)$ bounded by zero-flux surfaces ${ }^{3}$ possess unambiguous energies and energy-mediated properties [1] — necessary for checking results against measured/predicted values, and for making contact with the broader physical sciences. For any system there exists in $\rho$ an

\footnotetext{
1 Department of Chemistry, Colorado School of Mines, Golden CO, USA

2 twilson@mines.edu

${ }^{3}$ Surfaces through which the flux of electron density gradient is everywhere zero
} 
infinite number of such regions, which are called gradient bundles and can be constructed arbitrarily [2]. This infinite set is elegantly reduced by appealing to the topology of $\rho$, which precisely defines a finite set of regions that correspond to atoms in molecules (atomic basins) $[1,3]$. Alternatively, $\rho$ can be infinitesimally decomposed using differential gradient bundles (GBs), producing the gradient bundle condensed charge density $(\mathcal{P})$. The topology of $\mathcal{P}$ uniquely defines a second set of regions that correspond to chemical bonds (bond bundles) or non-bonding regions (e.g. lone pairs) $[2,4]$.

Either type of $\rho$ partitioning, into atomic basins or bond bundles, produces a set of nonoverlapping regions that combine to fill all space, along with a set of condensed regional properties - integrated over each region — such as atomic (or bond) volume, energy, and electronic population (electron count). Comparing atomic condensed properties across chemical systems, one can inspect the property flow between atoms that results from whatever perturbation was applied to the non-reference system. Likewise, comparing bond bundle condensed properties, one can inspect the property flow within and between bonds, and between the particular atomic regions (bond "wedges") participating in a bond [5]. Taken together, the sets of atomic and bond condensed properties provide a qualitative and quantitative comparison tool for investigating any number of chemical and materials science phenomena.

Here, we'll partially reproduce the investigation by Meir et al. into Diels-Alder catalysis and selectivity using oriented electric fields [6], which is an increasingly important area of study especially as it pertains to enzymatic catalysis [7-10], using the combined toolset of atomic basin/bond bundle decompositions. Our investigation will be limited to the catalytic effect. We'll also see how $\mathcal{P}$ and other property fields computable using a gradient bundle decomposition enable the direct inspection of electron density, volume, and energy redistribution within an atom, and how this can be used to reveal the subtle changes in structure that underly the catalytic effects of the applied electric field.

\section{Background}

\section{Gradient bundle decomposition}

To paraphrase our earlier article [2], the field $\mathcal{P}$ is constructed by mapping points in $\mathcal{P}$ to electron density gradient $(\nabla \rho)$ paths. The origin and terminus of a gradient path $(\mathrm{G})$ are 
respectively a local minimum called a cage critical point $(\mathrm{CP})$ — which may be at $\infty$ —and a local maximum typically coincident with a nucleus and thus dubbed a nuclear CP. Each G is also parameterized by arclength $(s)$.

All Gs are radial as they approach their terminuses, allowing one to define a spherical polar coordinate system about each nuclear CP so that each of the $\infty$ Gs in $\rho$ may be uniquely specified by its terminating nuclear $\mathrm{CP}$ index and the polar and azimuthal angles at which it terminates, i.e. $\mathrm{G}_{i}(\theta, \phi)$. Thus the independent variables of $\rho$ within an atomic basin $i$ are converted from $(x, y, z)$ to $(\theta, \phi, s)$.

With the atomic gradient reference system established, we can conceptually perform a gradient bundle decomposition by first placing a sphere $S_{i}$ of radius $d r$ (in practice, $d r \lesssim$ $0.2 \AA$ ) centered at nuclear CP $i$. We then partition the surface of $S_{i}$ into differential area elements $d A=d r^{2} d \theta d \phi$ (see Figure 1). The Gs intersecting $S_{i}$ interior to a particular area element constitute a differential volume element, bounded by zero-flux surfaces and called a differential gradient bundle, $d \mathrm{~GB}_{i}(\theta, \phi)$. Importantly, the cross-sectional area of each $d \mathrm{~GB}_{i}$ itself varies with arclength, i.e. $d A=d A(s)$. The union of all $d \mathrm{~GB}_{i}$ recovers the atomic basin, while $d \mathrm{~GB}_{i}$ are the smallest structures bounded by zero-flux surfaces, hence the smallest structures possessing well-defined energies and energy-mediated

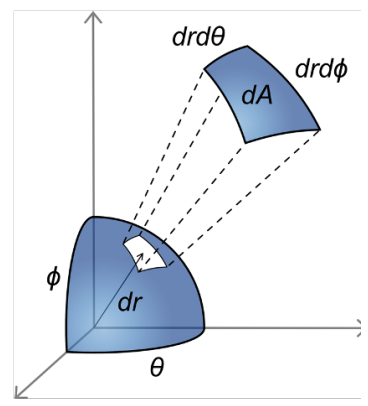

Figure 1. A differential area element on a sphere. properties.

\section{The condensed charge density and bond bundles}

By mapping the integrated $\rho$ content of each $d \mathrm{~GB}_{i}(\theta, \phi)$ to its corresponding position at $S_{i}(\theta, \phi)$, we produce the gradient bundle condensed charge density, $\mathcal{P}_{i}(\theta, \phi)$, which has units of electrons per area, specifically electrons per steradian $(s r)$. Additionally, for any atomic scalar property field, $F_{i}$, there exists a corresponding gradient bundle condensed scalar field, $\mathcal{F}_{i}$, that is a function of $\theta$ and $\phi$ and a functional of $F_{i}$, such that,

$$
\mathcal{F}\left[F_{i}\right] \equiv \mathcal{F}_{i}\left[\theta, \phi, F_{i}(\theta, \phi, s)\right]=\int_{\mathrm{G}_{i}(\theta, \phi)} F_{i}(s) d A(s) d s
$$

Using $\rho$ as the input function, a GB decomposition yields the condensed charge density $(\mathcal{F}[\rho]=\mathcal{P})$, the (gradient or Laplacian forms of) kinetic energy density yields the condensed 

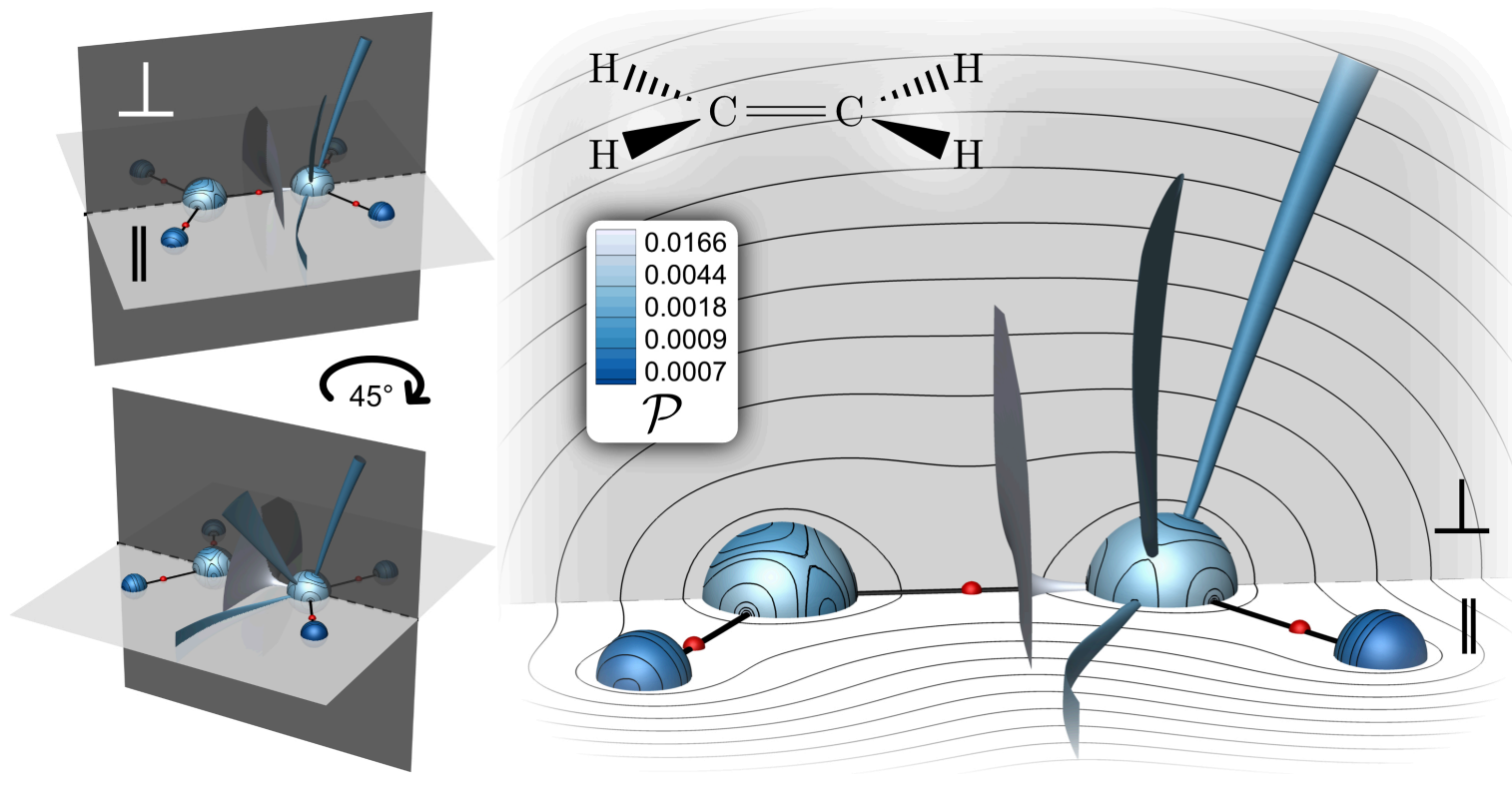

Figure 2. The condensed density $\mathcal{P}$ mapped as contours onto spheres centered at the $\mathrm{C}$ and $\mathrm{H}$ nuclei in ethylene. Gradient bundles corresponding to the maximum, minimum, and saddle CPs-intersecting the sphere along equiradial CP-centered circles-are shown for a $\mathrm{C}$ atom. Contours of $\rho$ are shown in the molecular plane $\|$ and the perpendicular, $C=C$ bond axis coincident plane $\perp$.

kinetic energy density $\left(\mathcal{F}\left[T_{G}\right]=\mathcal{F}\left[T_{L}\right]=\mathcal{T}\right)$, etc. If the constant function $F_{i}(\theta, \phi, s)=1$ is used as input, the gradient bundle condensed volume is produced $(\mathcal{F}[1]=\mathcal{V}),{ }^{4}$ which also corresponds to the integrated Gaussian curvature of charge density isosurfaces within each gradient bundle $[2,5]$.

An algorithmic GB decomposition looks quite similar, using a finite number of $d \mathrm{~GB}_{i}$-at approximately 6,000 per atom for this demonstration-bounded by Gs intersecting at and along the nodes and edges of a triangulated sphere mesh. Gaussian quadrature tetrahedral numerical integration is performed for each GB by decomposing it, first into convex polyhedra defined by the GB intersections with lower and upper $\rho$ isosurfaces, and again into tetrahedra. The resulting integrals are mapped to the midpoint of each GB's corresponding triangular sphere element and visualized as contours on the sphere mesh surface.

By virtue of its construction, a point in $\mathcal{P}$ maps to a $G$ in $\rho$, any arbitrary path in $\mathcal{P}$ maps to a zero-flux surface, and any closed path in $\mathcal{P}$ corresponds to a gradient bundle in $\rho$. Each sphere mapping of $\mathcal{P}$ (and other field variables) is called an atomic chart, and the collection of all atomic charts gives the system (molecular, crystalline, etc.) atlas.

${ }^{4}$ In open systems, a step function-defined to be one within some truncating isosurface (typically $\rho=$ $0.001 \mathrm{au}$ ) and zero beyond-is used to calculate $\mathcal{V}$. 


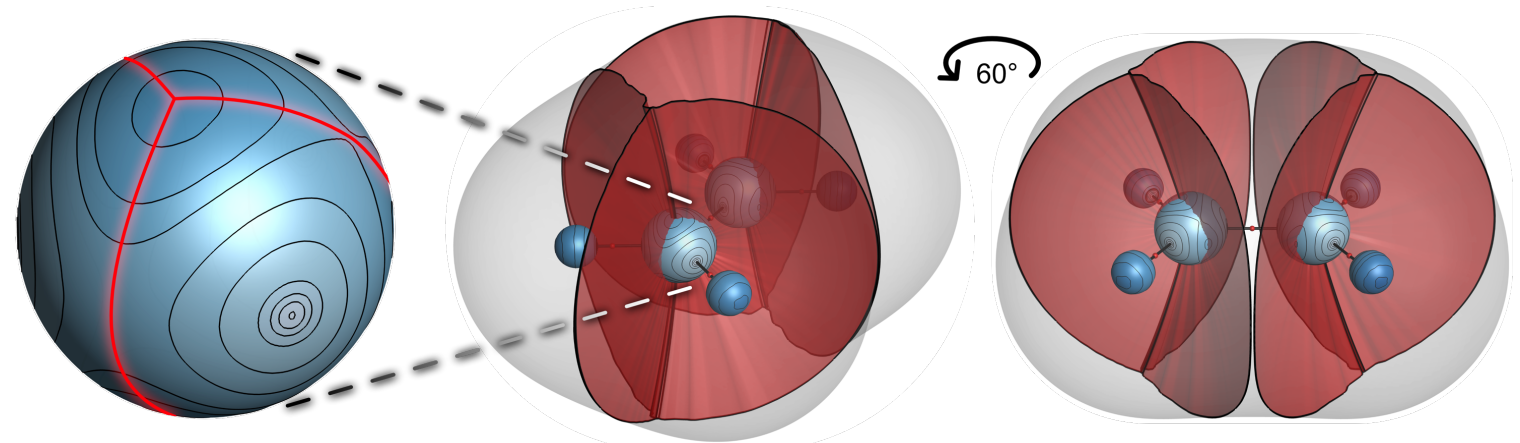

Figure 3. The three carbon maximum basins in ethylene and their corresponding bond bundle surfaces.

The critical points of $\mathcal{P}$ include maxima, minima, and saddle points. Figure 2 shows $\mathcal{P}$ mapped onto nuclei-centered spheres in ethylene, along with representative gradient bundles that correspond to $\mathcal{P}$ CPs. Often, bond paths map to maxima, as in ethylene where the black bond paths exclusively intersect atomic charts at local maxima. This topologically partitions $\mathcal{P}$ in a way analogous to the Bader atoms. In $\rho$, the union of all Gs sharing a common terminal nuclear CP specifies a unique volume - the atomic basin. Similarly, the union of gradient paths $(\mathcal{G})$ through $\mathcal{P}$ sharing a common terminal maximum specifies a unique areaa maximum basin — corresponding to a unique gradient bundle called a bond wedge. When bond wedges on neighboring atoms share some portion of an interatomic surface, they combine to form a bond bundle. Figure 3 depicts the bond wedges of a $\mathrm{C}$ atom in ethylene, delineated by red $\mathcal{G}$ linking saddle to minimum $\mathrm{CPs}$, and the corresponding ethylene bond bundle surfaces.

Bond bundles possess a number of qualitative and quantitative properties, many of which we have only recently developed the ability to calculate and analyze. ${ }^{5}$ Early in bond bundle research, it was found that their valance electron population recovers bond order [11], total electron population corresponds to bond strength [12], and that the shapes of their surfaces correspond to chemical functionality and can indicate molecular regions prone to nucleophilic or electrophilic attack [13]. The topology of the gradient bundle condensed charge density recovers the same bond bundle surfaces as the previous method [4], but with higher accuracy and generality to more chemical and material systems.

5 Previously, bond bundles were analyzed using a method of explicit bond bundle surface identification, rather than implicit identification using the gradient bundle condensed charge density as at present. 


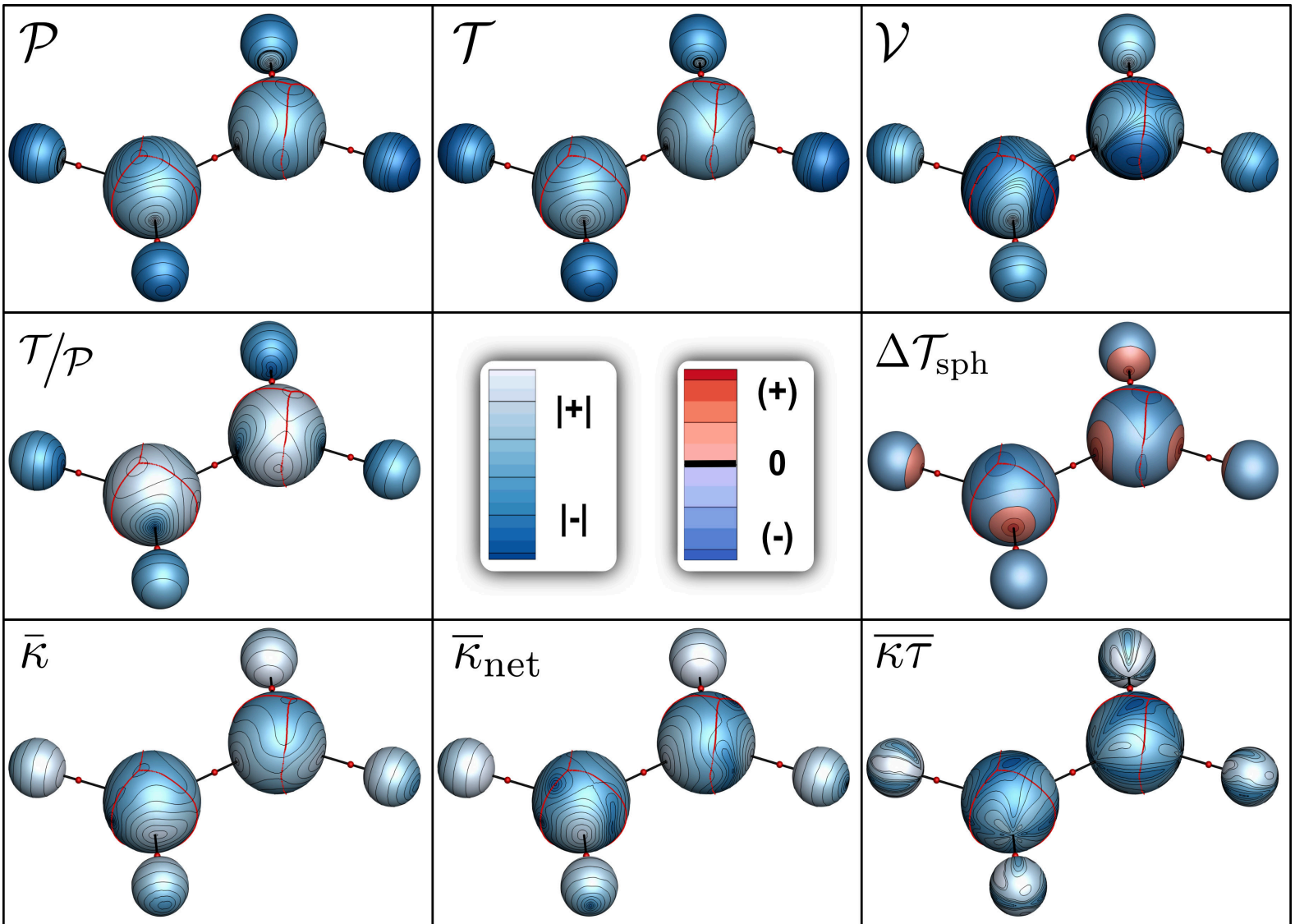

Figure 4. Gradient bundle condensed scalar properties (top row), derived properties (middle row), and geometric charge density descriptors (bottom row) shown as contours mapped onto spheres around the nuclei of ethylene. Values are everywhere positive and are shaded blue to white to indicate low to high values, except for $\Delta \mathcal{T}_{\text {sph }}$, where blue and red indicate negative and positive values. Red lines on carbon atom spheres indicate the boundaries between maximum basins defined according to $\mathcal{P}$. 


\section{Visual qualitative inspection of gradient bundle condensed properties}

Figure 4 shows a selection of condensed property fields as contours mapped onto the atoms of ethylene. The top, middle, and bottom rows respectively show condensed properties from each category. Starting with the condensed scalar fields (category A; top row), in organic systems the condensed density $(\mathcal{P})$ primarily governs the topology of the condensed kinetic energy $(\mathcal{T})$ and volume $(\mathcal{V})$, that is, they typically have same sets of maximum, minimum, and saddle CPs $[2,4]$. Importantly, this means that, in many systems, bond bundles, defined according to $\mathcal{P}$ (see the red paths on the spheres in Figure 4), wouldn't be dramatically different if defined according to another condensed scalar property such as kinetic energy density.

$\mathcal{V}$, however, is also a measure of total geometric Gaussian curvature within gradient bundles [2,5], and can deviate from the behavior of $\mathcal{P}$ more so than $\mathcal{T}$. In this case, note that on the carbon atoms, $\mathcal{P}$ and $\mathcal{T}$ each have four minimum CPs, one each above and below the molecular plane, and one each within the molecular plane on either side of the $\mathrm{C}=\mathrm{C}$ bond. In $\mathcal{V}$, however, there is an additional minimum $\mathrm{CP}$ on the "back" of the carbon atom at the $\mathrm{H}-\mathrm{C}-\mathrm{H}$ position. This character is also reflected in the geometric descriptors (category $\mathrm{C}$; bottom row) in the average gradient bundle curvature $(\bar{\kappa})$ and average net gradient bundle curvature $\left(\bar{\kappa}_{\text {net }}\right)$ that also have "extra" minimum CPs in the same region on the C atoms; confirmation of the link between condensed volume and charge density geometric curvature. The average curvature-scaled gradient bundle torsion ${ }^{6}(\overline{\kappa \tau})$ shows, at a glance, which gradient paths within an atomic basin will bend and curve primarily within the same plane. Here we see that gradient paths within the carbon atomic basins will have very low torsion if they are close to the molecular plane or the perpendicular plane - see the minimum CP representative gradient bundles in Figure 2-and that those at approximately $45^{\circ}$ to both planes will achieve maximum torsion - see the saddle CP gradient bundle in the same figure. This is to be expected, since both planes are symmetry planes and hence zero flux surfaces. The same general behavior is also observed, however, on the $\mathrm{H}$ atoms, not only in the molecular plane, but in the plane perpendicular to the molecular plane and also containing a $\mathrm{C}-\mathrm{H}$ bond axis.

${ }^{6} \bar{\kappa}$ is computed as the gradient path integral of the angles between neighboring line segments along a discretized path, $\bar{\kappa}_{\text {net }}$ is computed as the angle between the originating and terminal ends of a gradient path, and $\overline{\kappa \tau}$ is computed as the gradient path integral of the of angles between planes defined by coincident pairs of line segments (three neighboring line segments, where the central segment is shared between the pairs) along the path, scaled at each line segment by the its value of path curvature. All three properties are computed for paths and then averaged over the gradient paths defining a particular gradient bundle to recover its values. 
Investigations into the chemical significance of these pure geometric descriptors are ongoing, but preliminary results show that the distribution and redistribution of charge density curvature plays an important role in such fundamental chemical processes as carbonyl bond activation [5].

Turning to the middle row of Figure 4, there are two derived condensed properties shown. The first is average kinetic energy per electron $(\mathcal{T} / \mathcal{P})$, which has been investigated by Morgenstern et al. using another type of gradient bundle decomposition applicable to systems with linear symmetry [15] (see chapter \#\#\#). Unlike $\mathcal{P}$ and $\mathcal{T}$, that typically achieve maxima at bond paths, the kinetic energy per electron has minimum CPs at bond paths. This is at least the case for covalent bonds, where electrons accumulate in the inter-atomic region, thus lowering their kinetic energy relative to an atomic system where they orbit closer to the nucleus. Maximum kinetic energy per electron is then achieved away from bonding regions where the charge density can remain contracted near the nucleus with higher kinetic (and offsetting potential) energy. Note also that that the minimum basins of $\mathcal{T} / \mathcal{P}$ are nearly identical to the bonding regions defined by $\mathcal{P}$, providing another viable alternative way to define the boundaries between bonding regions [15].

The second derived condensed property shown in Figure 4 is the condensed deformation kinetic energy density $\left(\Delta \mathcal{T}_{\mathrm{sph}}\right)$. All condensed deformation properties are calculated in the same way, by subtracting from the condensed property field, the equivalent field in a spherical atomic reference state,

$$
\Delta \mathcal{F}_{\text {sph }}=\mathcal{F}-\mathcal{F}_{\text {sph }}
$$

That is, comparing the value at a point to what the value would have been if the total atomic basin condensed value in the current system were distributed with spherical (atomic) symmetry, hence "equivalent". Each differential gradient bundle's atomic reference state value is simply its share of the atomic basin condensed value as determined by its normalized solid angle $(\alpha)$, so the value of some condensed deformation property for differential gradient bundle $i$ is

$$
\Delta \mathcal{F}_{\mathrm{sph}, i}=\mathcal{F}_{i}-\alpha_{i} F_{\text {atom }},
$$

where $F_{\text {atom }}$ is the atomic basin condensed property value. In Figure 4 we see that kinetic energy accumulates at and near bond paths, and that there is a precise boundary separating these regions from those of kinetic energy depletion. The $\Delta \mathcal{T}_{\text {sph }}=0$ contour (separating blue from red in Figure 4), like all closed paths through gradient bundle condensed property fields, specifies a precise zero-flux surface bounded region in $\rho$. Due to the virial theorem, we know that within regions bounded by zero-flux surfaces the total regional kinetic energy is 
Table 1. Atomic basin, bond wedge, and bond bundle condensed properties in ethylene. All gradient bundles are truncated at the $\rho=0.001$ au isosurface $(98.9 \%$ of electrons recovered).

\begin{tabular}{|c|c|c|c|c|c|c|c|c|c|c|}
\hline Region & $\begin{array}{c}\rho \\
{[\mathrm{e}]} \\
\end{array}$ & $\begin{array}{c}T \\
{[\mathrm{Ha}]} \\
\end{array}$ & $\begin{array}{c}V \\
{\left[a_{0}^{3}\right]} \\
\end{array}$ & $\begin{array}{c}\bar{\kappa} / V \\
{\left[\mathrm{rad} / a_{0}^{3}\right]} \\
\end{array}$ & $\begin{array}{l}\bar{\kappa}_{\text {net }} / V \\
{\left[\mathrm{rad} / a_{0}^{3}\right]} \\
\end{array}$ & $\begin{array}{c}\kappa \tau / V \\
{\left[\mathrm{rad} / a_{0}^{3}\right]} \\
\end{array}$ & $\begin{array}{c}T / \rho \\
{[\mathrm{Ha} / \mathrm{e}]} \\
\end{array}$ & $\begin{array}{r}\Delta T_{\mathrm{sph}} \\
{[\Delta \mathrm{Ha}]} \\
\end{array}$ & $\begin{array}{c}\Delta T_{\mathrm{sph}}^{-} \\
{[\Delta \mathrm{Ha}]} \\
\end{array}$ & $\begin{array}{c}\Delta T_{\mathrm{sph}}^{+} \\
{[\Delta \mathrm{Ha}]} \\
\end{array}$ \\
\hline C1 atomic basin & 6.032 & 5.993 & 98.51 & 10.100 & 5.957 & 0.328 & 0.994 & 0.000 & -0.723 & 0.723 \\
\hline \bond wedge & 2.441 & 2.432 & 38.43 & 9.307 & 6.707 & 0.325 & 0.996 & 0.068 & -0.270 & 0.338 \\
\hline 4 bond wedge & 1.787 & 1.773 & 29.84 & 10.082 & 5.168 & 0.321 & 0.992 & -0.033 & -0.224 & 0.190 \\
\hline 4 bond wedge & 1.804 & 1.788 & 30.24 & 11.125 & 5.783 & 0.339 & 0.991 & -0.035 & -0.229 & 0.195 \\
\hline $\mathrm{C} 2$ atomic basin & 6.032 & 5.993 & 98.52 & 9.938 & 5.863 & 0.324 & 0.994 & 0.000 & -0.723 & 0.723 \\
\hline 4 bond wedge & 2.442 & 2.431 & 38.39 & 9.316 & 6.707 & 0.324 & 0.996 & 0.072 & -0.269 & 0.341 \\
\hline 4 bond wedge & 1.802 & 1.786 & 30.23 & 10.805 & 5.590 & 0.333 & 0.991 & -0.034 & -0.227 & 0.193 \\
\hline 4 bond wedge & 1.788 & 1.776 & 29.89 & 9.861 & 5.054 & 0.314 & 0.993 & -0.038 & -0.226 & 0.189 \\
\hline $\mathrm{H} 1$ atomic basin & 0.942 & 0.584 & 48.34 & 30.570 & 24.068 & 1.418 & 0.620 & 0.000 & -0.163 & 0.163 \\
\hline $\mathrm{H} 2$ atomic basin & 0.942 & 0.584 & 48.34 & 29.996 & 23.625 & 1.405 & 0.620 & 0.000 & -0.163 & 0.163 \\
\hline H3 atomic basin & 0.942 & 0.584 & 48.34 & 30.572 & 24.068 & 1.419 & 0.620 & 0.000 & -0.163 & 0.163 \\
\hline $\mathrm{H} 4$ atomic basin & 0.942 & 0.584 & 48.34 & 29.996 & 23.624 & 1.404 & 0.620 & 0.000 & -0.163 & 0.163 \\
\hline Total & 15.830 & 14.322 & 390.38 & 20.056 & 14.794 & 0.864 & 0.905 & 0.000 & -2.097 & 2.097 \\
\hline $\mathrm{C} 1-\mathrm{C} 2$ bond bundle & 4.883 & 4.863 & 76.83 & 9.311 & 6.707 & 0.325 & 0.996 & 0.139 & -0.540 & 0.679 \\
\hline \& C1 bond wedge & 2.441 & 2.432 & 38.43 & 9.307 & 6.707 & 0.325 & 0.996 & 0.068 & -0.270 & 0.338 \\
\hline \C2 bond wedge & 2.442 & 2.431 & 38.39 & 9.316 & 6.707 & 0.324 & 0.996 & 0.072 & -0.269 & 0.341 \\
\hline $\mathrm{C} 1-\mathrm{H} 2$ bond bundle & 2.729 & 2.357 & 78.18 & 22.395 & 16.580 & 0.991 & 0.864 & -0.033 & -0.386 & 0.353 \\
\hline ¿C1 bond wedge & 1.787 & 1.773 & 29.84 & 10.082 & 5.168 & 0.321 & 0.992 & -0.033 & -0.224 & 0.190 \\
\hline \H2 bond wedge & 0.942 & 0.584 & 48.34 & 29.996 & 23.625 & 1.405 & 0.620 & 0.000 & -0.163 & 0.163 \\
\hline $\mathrm{C} 1-\mathrm{H} 3$ bond bundle & 2.745 & 2.372 & 78.58 & 23.088 & 17.031 & 1.003 & 0.864 & -0.035 & -0.392 & 0.357 \\
\hline \C1 bond wedge & 1.804 & 1.788 & 30.24 & 11.125 & 5.783 & 0.339 & 0.991 & -0.035 & -0.229 & 0.195 \\
\hline \H3 bond wedge & 0.942 & 0.584 & 48.34 & 30.572 & 24.068 & 1.419 & 0.620 & 0.000 & -0.163 & 0.163 \\
\hline $\mathrm{C} 2-\mathrm{H} 1$ bond bundle & 2.744 & 2.370 & 78.57 & 22.966 & 16.959 & 1.000 & 0.864 & -0.034 & -0.390 & 0.356 \\
\hline \C2 bond wedge & 1.802 & 1.786 & 30.23 & 10.805 & 5.590 & 0.333 & 0.991 & -0.034 & -0.227 & 0.193 \\
\hline ¿ H1 bond wedge & 0.942 & 0.584 & 48.34 & 30.570 & 24.068 & 1.418 & 0.620 & 0.000 & -0.163 & 0.163 \\
\hline $\mathrm{C} 2-\mathrm{H} 4$ bond bundle & 2.730 & 2.360 & 78.23 & 22.302 & 16.528 & 0.988 & 0.865 & -0.038 & -0.389 & 0.351 \\
\hline \C2 bond wedge & 1.788 & 1.776 & 29.89 & 9.861 & 5.054 & 0.314 & 0.993 & -0.038 & -0.226 & 0.189 \\
\hline $4 \mathrm{H} 4$ bond wedge & 0.942 & 0.584 & 48.34 & 29.996 & 23.624 & 1.404 & 0.620 & 0.000 & -0.163 & 0.163 \\
\hline Total & 15.830 & 14.322 & 390.38 & 20.056 & 14.794 & 0.864 & 0.905 & 0.000 & -2.097 & 2.097 \\
\hline
\end{tabular}

equal and opposite the total energy [16,17]. So, in this case, the regions in $\rho$ specified by the $\Delta \mathcal{T}_{\mathrm{sph}}=0$ contour are the regions of total energetic stabilization and countervailing destabilization in ethylene's ground state.

Condensed deformation properties may also be calculated by providing a reference value to use in place of $F_{\text {atom }}$. For example, if an accurate and comparable atomic energy is already known, it may be used to define the spherical atomic reference state.

\section{Quantitative condensed property analysis}

Table 1 lists the property fields from Figure 4, condensed over atomic basins, bond wedges, and bond bundles, where $\mathrm{C}$ bond wedge values correspond to the regions delineated by red paths in Figure 4. Note that it is known that the primary source of error in the current 
implementation arises from interpolation error from the exclusive use of regular volumetric property grids. Starting again with gradient bundle condensed scalar fields, the current gradient bundle decomposition method recovers accurate atomic basin populations and energies, with agreement between symmetry-degenerate atoms to beyond a thousandth of an electron or Hartree $(\sim 0.6 \mathrm{kcal} / \mathrm{mol})$. Agreement between symmetry-degenerate bond wedges is less, to around a hundredth of an electron or Hartree. While the equivalent $\sim 60 \mathrm{kcal} / \mathrm{mol}$ would constitute an unacceptable amount of error in normal terms, here recall that these bond wedges, defined in the condensed $(\theta, \phi)$ space, coincide with a portion of an atomic nucleus, where electronic kinetic energies are highest. Thus a slight error in the identification of bond wedge boundaries results in a misallocation of some core energy and density. This error is being reduced as improvements are made to the underlying numerical methods for identifying the boundaries between bond wedges. In the meantime, because all gradient bundle condensed properties are additive, the atoms and regions known to be symmetry degenerate can be averaged to obtain a more accurate result, as done elsewhere in this work. Additionally, for condensed scalar properties, their corresponding condensed deformation property provides a more chemically relevant account of regional properties, such as with the deformation kinetic energy, $\Delta T_{\mathrm{sph}}$, where agreement between bond wedges is on the order of a thousandth of a Hartree.

When reporting regional condensed values for geometric descriptors they should be averaged over some other regional property. Here we've averaged over (divided by) volume, resulting in units of angle per volume that gives a clear sense of one volumetric region being more or less curved (i.e. less or more spherical; "atomic") than another.

We return briefly to $\Delta T_{\mathrm{sph}}$ which, by definition, integrates to zero over an atomic basinlike all deformation properties. Here the signed components of the function, $\Delta T_{\mathrm{sph}}^{-}$and $\Delta T_{\mathrm{sph}}^{+}$, can nonetheless be used to calculate the extent of intra-atomic redistribution of energy (or any condensed scalar property). $\Delta T_{\mathrm{sph}}$ is itself non-zero for subatomic regions, and Table 1 indicates that the $\mathrm{C}-\mathrm{H}$ bonds in ethylene are destabilized (lower kinetic energy thus higher total energy) and that the $\mathrm{C}=\mathrm{C}$ bond stabilized as a necessary result of bonding.

\section{Electric Field Charge Density Response and Catalysis}

Oriented electric fields have been demonstrated to influence chemical reactions in the 


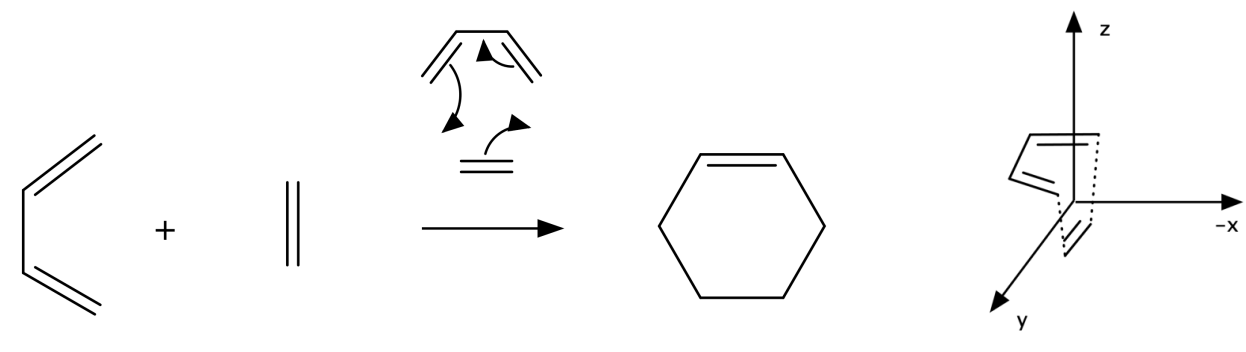

Scheme 2. The prototypical non-polar Diels-Alder reaction of butadiene and ethylene (left) and the orientation of the reactants (right; note the negation of the $\boldsymbol{x}$-axis).

laboratory and in biological systems [79,18-22], and are now thought to play a significant role in nature's ability to enzymatically catalyze reactions several orders of magnitude faster than artificial catalysts [23-26]. Deliberate reaction rate and selectivity control in the biologically and industrially significant Diels-Alder reaction has been experimentally achieved $[18,27,28]$ and theoretically predicted $[6,29]$, and as the broader field of electrostatic catalysis continues to progress, Diels-Alder reactions continue to provide conceptual insight.

In their theoretical investigation, Meir et al. modeled two Diels-Alder reactions with and without electric fields of varying sign and magnitude [6]. The first reaction was the cycloaddition of ethylene and cis-butadiene to form cyclohexene (see Scheme 2), where they found that an electric field oriented along the "reaction axis" pointing from the butadiene to the ethylene (the negative $z$ direction; electric field direction points positive to negative) lowered the reaction barrier, but that a field in the opposite direction did not raise the reaction barrier. Using this conventionally "simple" example of the charge density response to an electric field, we will examine the inter-atomic and intra-atomic redistribution of electron charge density that underly and accompany the response. 


\section{Computational Methods}

All DFT calculations, including those used to produce the energy and distance values in Scheme 1 were performed with the Amsterdam Modeling Suite [30-32] ab initio software using the Perdew-Burke-Ernzerhof (PBE) functional [33] and a triple-zeta with polarization (TZP) all-electron basis set. Implicit COSMO [34-36] solvation was applied to all simulations using Allinger solvent radii [37] and a dielectric constant of $\varepsilon=8.93$. All electric fields are of magnitude $0.0125 \mathrm{au}$ (approx. 64MV/cm). Analysis was performed within the Tecplot 360 visualization package [38] using the Gradient Bundle Decomposition software of the in house Bondalyzer package by the Molecular Theory Group at Colorado School of Mines [39].

The reaction of cis-butadiene and ethylene was modeled by bringing the cis-butadiene down on top of the ethylene, as depicted in the right side of Scheme 2. The resulting reaction profile is shown in Scheme 1 for the

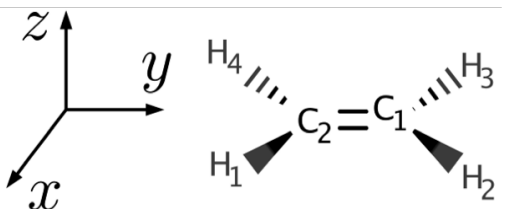

Scheme 3. Orientation of ethylene with respect to applied electric fields, and numbering of atoms.

Table 2. Atomic basin and bond bundle condensed charge densities in ethylene with and without 0.0125au applied electric fields oriented in the $x, y$, and $z$ directions.

\begin{tabular}{|c|c|c|c|c|c|c|c|c|c|c|}
\hline \multirow{3}{*}{ Region } & \multicolumn{10}{|c|}{$\rho[\mathrm{e}]$} \\
\hline & \multirow[t]{2}{*}{ NEF } & \multicolumn{3}{|c|}{ EEF $x$} & \multicolumn{3}{|c|}{ EEF $y$} & \multicolumn{3}{|c|}{ EEF $z$} \\
\hline & & & $\Delta$ & $\% \Delta$ & & $\Delta$ & $\% \Delta$ & & $\Delta$ & $\% \Delta$ \\
\hline C1 atomic basin & 6.032 & 6.032 & 0.000 & -0.004 & 5.988 & -0.044 & -0.723 & 6.033 & 0.001 & 0.013 \\
\hline C2 atomic basin & 6.032 & 6.032 & 0.000 & -0.003 & 6.076 & 0.045 & 0.741 & 6.032 & 0.001 & 0.011 \\
\hline $\mathrm{H} 1$ atomic basin & 0.942 & 0.891 & -0.050 & -5.337 & 0.987 & 0.046 & 4.838 & 0.941 & -0.001 & -0.088 \\
\hline $\mathrm{H} 2$ atomic basin & 0.942 & 0.891 & -0.050 & -5.346 & 0.895 & -0.047 & -4.956 & 0.941 & -0.001 & -0.060 \\
\hline $\mathrm{H} 3$ atomic basin & 0.942 & 0.992 & 0.050 & 5.316 & 0.895 & -0.047 & -4.950 & 0.941 & -0.001 & -0.091 \\
\hline $\mathrm{H} 4$ atomic basin & 0.942 & 0.991 & 0.050 & 5.298 & 0.987 & 0.045 & 4.820 & 0.941 & -0.001 & -0.061 \\
\hline Total & 15.830 & 15.829 & -0.001 & -0.007 & 15.829 & -0.001 & -0.008 & 15.829 & -0.001 & -0.009 \\
\hline $\mathrm{C} 1-\mathrm{C} 2$ bond bundle & 4.883 & 4.883 & 0.000 & 0.003 & 4.906 & 0.024 & 0.489 & 4.859 & -0.023 & -0.475 \\
\hline$\measuredangle C 1$ bond wedge & 2.441 & 2.453 & 0.012 & 0.505 & 2.265 & -0.176 & -7.217 & 2.432 & -0.009 & -0.355 \\
\hline \C2 bond wedge & 2.442 & 2.429 & -0.012 & -0.500 & 2.642 & 0.200 & 8.194 & 2.427 & -0.015 & -0.594 \\
\hline $\mathrm{C} 1-\mathrm{H} 2$ bond bundle & 2.729 & 2.770 & 0.041 & 1.506 & 2.741 & 0.013 & 0.466 & 2.735 & 0.007 & 0.243 \\
\hline \C1 bond wedge & 1.787 & 1.879 & 0.091 & 5.116 & 1.846 & 0.059 & 3.323 & 1.794 & 0.007 & 0.402 \\
\hline $4 \mathrm{H} 2$ bond wedge & 0.942 & 0.891 & -0.050 & -5.346 & 0.895 & -0.047 & -4.956 & 0.941 & -0.001 & -0.060 \\
\hline $\mathrm{C} 1-\mathrm{H} 3$ bond bundle & 2.745 & 2.691 & -0.054 & -1.965 & 2.772 & 0.027 & 0.968 & 2.747 & 0.001 & 0.050 \\
\hline \C1 bond wedge & 1.804 & 1.700 & -0.104 & -5.766 & 1.877 & 0.073 & 4.058 & 1.806 & 0.002 & 0.124 \\
\hline$\measuredangle H 3$ bond wedge & 0.942 & 0.992 & 0.050 & 5.316 & 0.895 & -0.047 & -4.950 & 0.941 & -0.001 & -0.091 \\
\hline $\mathrm{C} 2-\mathrm{H} 1$ bond bundle & 2.744 & 2.783 & 0.039 & 1.429 & 2.714 & -0.029 & -1.063 & 2.746 & 0.002 & 0.081 \\
\hline$\measuredangle C 2$ bond wedge & 1.802 & 1.891 & 0.089 & 4.965 & 1.727 & -0.075 & -4.147 & 1.805 & 0.003 & 0.169 \\
\hline$\measuredangle H 1$ bond wedge & 0.942 & 0.891 & -0.050 & -5.337 & 0.987 & 0.046 & 4.838 & 0.941 & -0.001 & -0.088 \\
\hline $\mathrm{C} 2-\mathrm{H} 4$ bond bundle & 2.730 & 2.702 & -0.028 & -1.010 & 2.695 & -0.035 & -1.291 & 2.741 & 0.012 & 0.424 \\
\hline \C2 bond wedge & 1.788 & 1.711 & -0.077 & -4.331 & 1.708 & -0.081 & -4.509 & 1.800 & 0.012 & 0.679 \\
\hline $4 \mathrm{H} 4$ bond wedge & 0.942 & 0.991 & 0.050 & 5.298 & 0.987 & 0.045 & 4.820 & 0.941 & -0.001 & -0.061 \\
\hline Total & 15.830 & 15.829 & -0.001 & -0.007 & 15.829 & -0.001 & -0.008 & 15.829 & -0.001 & -0.009 \\
\hline
\end{tabular}


reaction with no applied electric field (NEF). Optimized geometries were obtained for all four steps and used for single point calculations with the various oriented electric fields.

\section{Results and Discussion}

\section{Ethylene electric field response}

Our analysis of the electric field response of the reaction starts with a lone reactant ethylene molecule (Scheme 3) and three 0.0125au applied external electric fields (EEFs) oriented orthogonally along the $\mathrm{C}=\mathrm{C}$ bond axis $(y$, pointing from $\mathrm{C} 2$ to $\mathrm{C} 1$ ), in the molecular plane $(x)$, and out of the molecular plane $(z)$. Table 2 lists regional condensed electron counts for ethylene with no electric field (NEF) and for each of the oriented applied fields, along with their difference $\left(\rho_{\mathrm{EEF}}-\rho_{\mathrm{NEF}}\right)$ and percent difference $\left(\rho_{\mathrm{EEF}}-\rho_{\mathrm{NEF}}\right) / \rho_{\mathrm{NEF}} \times 100$. Starting with the atomic basins, we see overall inter-atomic charge transfer of around a twentieth of an electron for the $x$ and $y$ fields and negligible transfer for the $z$ field to which the molecule is symmetry invariant. The direction of $x$ and $y$ charge transfer is as expected for a homogeneous electron gas; opposite that of the field.

For the $x$ field, charge density is "pushed" from $\mathrm{H} 1$ to $\mathrm{H} 4$ and from $\mathrm{H} 2$ to $\mathrm{H} 3$, leaving the $\mathrm{C}$ atoms unchanged, which makes sense as the $\mathrm{C}-\mathrm{H}$ bonds are identical, so an equal and

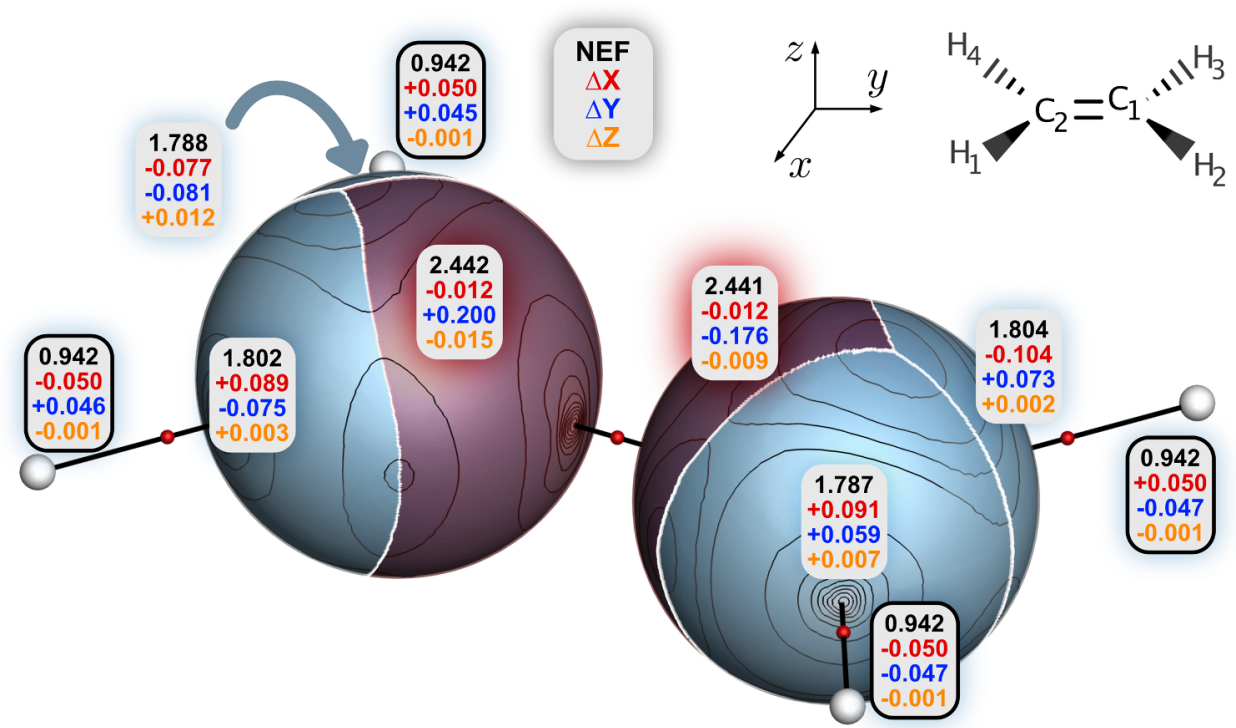

Figure 5. $\mathcal{P}$ maximum basins with their values of and changes in regional charge density in neutral ethylene (NEF) in response to electric fields oriented along the $x, y$, and $z$ directions. Basins are indicated by shading and separated by white borders. Red and grey shading indicate the $\mathrm{C}=\mathrm{C}$ and $\mathrm{C}-\mathrm{H}$ bonding regions respectively. 
opposite response is not surprising. Based on symmetry we can conclude that each $\mathrm{C}$ atom must have donated and accepted the same amount of density from and to its bonding $\mathrm{H}$ atoms, essentially left unchanged by the field. The $y$ field results pose something of an interpretative dilemma. They could be interpreted as another perfect example of electron density responding as an inhomogeneous electron gas if only homonuclear interactions are considered; charge flows from $\mathrm{H} 2$ to $\mathrm{H} 1$, from $\mathrm{H} 3$ to $\mathrm{H} 4$, and from $\mathrm{C} 1$ to $\mathrm{C} 2$, all by nearly equal amounts. Alternatively, the charge flow could be pictured as occurring between $\mathrm{CH}_{2}$ groups; from the $\mathrm{H} 2-\mathrm{C} 1-\mathrm{H} 3$ group to the $\mathrm{H} 1-\mathrm{C} 2-\mathrm{H} 4$ group. But when the interactions between bonded atoms are considered, the picture is less clear; $\mathrm{C} 1$ loses 0.044 net electrons, but gains 0.047 electrons from each hydrogen. Clearly, the intuition from a simple electron gas approximation to how the charge density should move in response to an electric field is quickly complicated by the presence of nuclear point charges. To see how the charge density response to an electric field is distributed among $\mathrm{C}-\mathrm{H}$ bonds relative to each other and the $\mathrm{C}=\mathrm{C}$ bond, we turn to analysis of subatomic bonding regions.

Inspection of the ethylene maximum basin (bond wedge) values in Figure 5 reveals a more intricate relationship between the charge redistribution to, from, and within $\mathrm{C}$ atoms. Recall that at the atomic scale the $x$-oriented field appeared to push charge through the $\mathrm{C} 1$ atom from $\mathrm{H} 2$ to $\mathrm{H} 3$, that is, $\mathrm{C} 1$ accepting charge from $\mathrm{H} 2$ and donating to $\mathrm{H} 3$, with the charge moving in the $-x$ direction. Here we see that $\mathrm{C} 1$ internally shifted charge in the opposite direction, from the $\mathrm{C} 1-\mathrm{H} 3$ bond wedge to the $\mathrm{C} 1-\mathrm{H} 2$ bond wedge, by an amount equal to that transferred to/from the $\mathrm{H}$ atoms; $\sim 0.05$ e gained from $\mathrm{H} 2, \sim 0.05$ e given to $\mathrm{H} 3$, and $\sim 0.05$ e shifted from the $\mathrm{C} 1-\mathrm{H} 3$ bond wedge to the $\mathrm{C} 1-\mathrm{H} 2$ bond wedge. The ambiguous case of the $y$-field response is seen here to be the same behavior. Within both $\mathrm{C}$ atoms, the intra-atomic charge 
redistribution is in the positive $y$-direction, opposite the direction of inter-atomic redistribution and opposite that expected of an electron gas.

That this charge shift is the opposite of the Coulombic response indicates that charge redistribution within the $\mathrm{C}$ atoms is a secondary electric-field response. The primary response is in the Van der Waals region of the molecule where the charge density is flatter with fewer changes in the sign of its geometric curvature. We previously reported how the fundamental geometric constraints on the shape of $\rho$ dictate that total Gaussian curvature over a closed charge density isosurface is constant (equal to $4 \pi$ ), and that increases in curvature in one region must be offset by decreases in curvature elsewhere [5]. We have also shown that, especially in organic systems, the gradient bundle condensed volume (a measure of total curvature) and density are topologically similar, so curvature and density

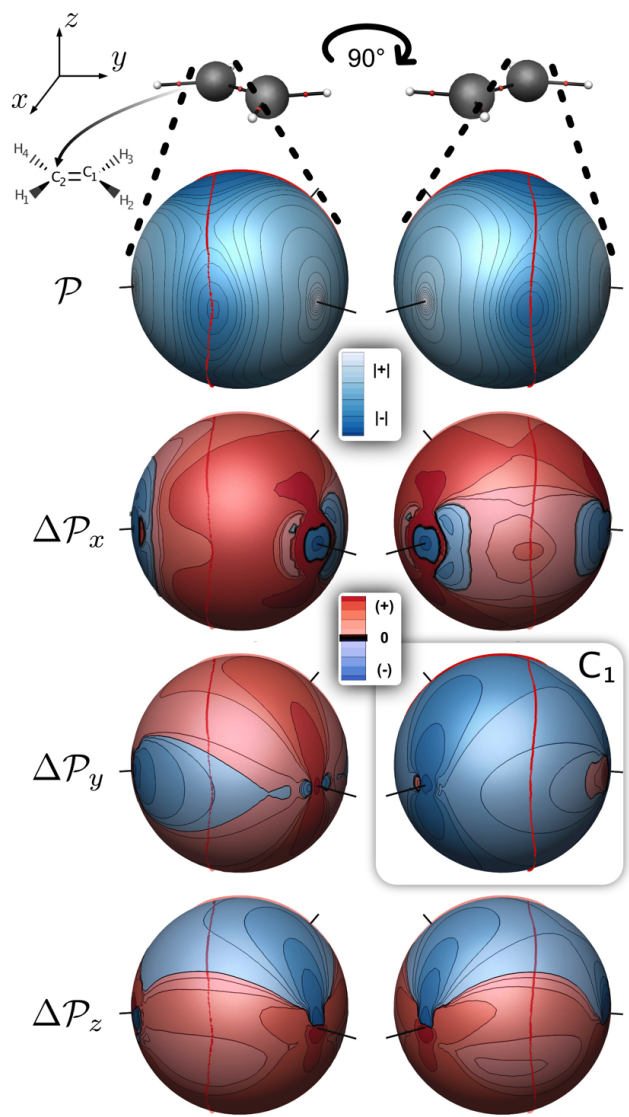

Figure 6. Difference condensed densities for the $\mathrm{C} 2$ atom in ethylene resulting from applied oriented electric fields. Maximum basin boundaries are overlaid as red lines. The columns show the same $\mathrm{C} 2$ atom, from angles $90^{\circ}$ apart, except for $\Delta \mathcal{P}_{y}$ where the right column shows the $\mathrm{C} 1$ atom. appear to flow in the same direction [2]. Thus the electron-gas-like behavior of $\rho$ in low-lying regions propagates into the molecule, constraining intra-atomic electron charge redistribution in a process akin to chemical induction [5].

We can also visually inspect the condensed charge density response to the electric fields by computing a difference condensed density, $\Delta \mathcal{P}$, similar in concept to the deformation kinetic energy density in Figure 4, but now instead of a spherical atomic reference state, the difference is that between the no-electric-field system and those with applied fields, $\Delta \mathcal{P}_{\mathrm{EEF}}=$ $\mathcal{P}_{\mathrm{EEF}}-\mathcal{P}_{\mathrm{NEF}}$. Figure 6 shows the difference densities corresponding to the ethylene $\mathrm{C}$ atomic response to the electric fields. There are clear regions of maximum accumulation and depletion resulting from the fields, and no bonding region experiences exclusively one or the 
Table 3. Atomic basin and bond bundle regional valence charge densities in the cis-butadiene + ethylene transition state with and without 0.0125au electric fields oriented along the $\pm z$ directions. Symmetrydegenerate atoms have been combined as indicated parenthetically

\begin{tabular}{|c|c|c|c|c|c|c|c|}
\hline \multirow{3}{*}{ Region } & \multicolumn{7}{|c|}{$\rho_{v}[e]$} \\
\hline & \multirow[t]{2}{*}{ NEF } & \multicolumn{3}{|c|}{$+z$ (ene to diene) } & \multicolumn{3}{|c|}{$-Z$ (diene to ene) } \\
\hline & & & $\Delta$ & $\% \Delta$ & & $\Delta$ & $\% \Delta$ \\
\hline C1 (C4) atomic basin & 4.074 & 4.064 & -0.010 & -0.234 & 4.087 & 0.013 & 0.324 \\
\hline C2 (C3) atomic basin & 4.036 & 3.995 & -0.042 & -1.028 & 4.082 & 0.046 & 1.133 \\
\hline C5 (C6) atomic basin & 4.050 & 4.081 & 0.031 & 0.777 & 4.022 & -0.028 & -0.680 \\
\hline $\mathrm{H} 1(\mathrm{H} 2)$ atomic basin & 0.953 & 0.923 & -0.030 & -3.138 & 0.980 & 0.027 & 2.851 \\
\hline $\mathrm{H} 3(\mathrm{H} 5)$ atomic basin & 0.944 & 0.941 & -0.003 & -0.325 & 0.944 & 0.000 & -0.006 \\
\hline $\mathrm{H} 4 \mathrm{H6}$ ) atomic basin & 0.954 & 0.943 & -0.011 & -1.150 & 0.963 & 0.009 & 0.985 \\
\hline $\mathrm{H} 7(\mathrm{H} 8)$ atomic basin & 0.948 & 0.983 & 0.035 & 3.641 & 0.911 & -0.037 & -3.882 \\
\hline H10 (H9) atomic basin & 0.948 & 0.975 & 0.027 & 2.840 & 0.918 & -0.030 & -3.190 \\
\hline Total & 33.814 & 33.809 & -0.004 & -0.012 & 33.815 & 0.002 & 0.005 \\
\hline $\mathrm{C} 1-\mathrm{C} 2$ bond bundle & 2.981 & 2.931 & -0.050 & -1.682 & 2.937 & -0.044 & -1.487 \\
\hline \C1 bond wedge & 1.534 & 1.518 & -0.016 & -1.030 & 1.487 & -0.047 & -3.074 \\
\hline$\measuredangle C 2$ bond wedge & 1.448 & 1.413 & -0.034 & -2.373 & 1.450 & 0.003 & 0.195 \\
\hline $\mathrm{C} 1-\mathrm{C} 5$ bond bundle & 0.312 & 0.356 & 0.044 & 14.004 & 0.299 & -0.013 & -4.278 \\
\hline \C1 bond wedge & 0.136 & 0.114 & -0.022 & -16.445 & 0.191 & 0.054 & 39.897 \\
\hline \C5 bond wedge & 0.176 & 0.242 & 0.066 & 37.645 & 0.108 & -0.068 & -38.574 \\
\hline $\mathrm{C} 1-\mathrm{H} 3$ bond bundle & 2.161 & 2.160 & -0.001 & -0.062 & 2.167 & 0.006 & 0.265 \\
\hline \C1 bond wedge & 1.217 & 1.219 & 0.002 & 0.142 & 1.223 & 0.006 & 0.476 \\
\hline \ H3 bond wedge & 0.944 & 0.941 & -0.003 & -0.325 & 0.944 & 0.000 & -0.006 \\
\hline $\mathrm{C} 1-\mathrm{H} 4$ bond bundle & 2.140 & 2.156 & 0.016 & 0.748 & 2.134 & -0.005 & -0.250 \\
\hline \C1 bond wedge & 1.186 & 1.213 & 0.027 & 2.273 & 1.171 & -0.015 & -1.242 \\
\hline$\measuredangle H 4$ bond wedge & 0.954 & 0.943 & -0.011 & -1.150 & 0.963 & 0.009 & 0.985 \\
\hline $\mathrm{C} 2-\mathrm{C} 3$ bond bundle & 2.785 & 2.672 & -0.113 & -4.045 & 2.817 & 0.032 & 1.150 \\
\hline \C2 bond wedge & 1.392 & 1.336 & -0.056 & -4.045 & 1.408 & 0.016 & 1.150 \\
\hline \C3 bond wedge & 1.392 & 1.336 & -0.056 & -4.045 & 1.408 & 0.016 & 1.150 \\
\hline $\mathrm{C} 2-\mathrm{H} 2$ bond bundle & 2.137 & 2.155 & 0.019 & 0.868 & 2.157 & 0.020 & 0.939 \\
\hline \C2 bond wedge & 1.183 & 1.232 & 0.048 & 4.095 & 1.176 & -0.007 & -0.602 \\
\hline$\triangle \mathrm{H} 2$ bond wedge & 0.953 & 0.923 & -0.030 & -3.138 & 0.980 & 0.027 & 2.851 \\
\hline $\mathrm{C} 5-\mathrm{C} 6$ bond bundle & 2.970 & 2.992 & 0.023 & 0.763 & 2.865 & -0.105 & -3.545 \\
\hline \C5 bond wedge & 1.485 & 1.496 & 0.011 & 0.763 & 1.432 & -0.053 & -3.545 \\
\hline \C6 bond wedge & 1.485 & 1.496 & 0.011 & 0.763 & 1.432 & -0.053 & -3.545 \\
\hline $\mathrm{C} 5-\mathrm{H} 8$ bond bundle & 2.142 & 2.137 & -0.005 & -0.226 & 2.174 & 0.032 & 1.504 \\
\hline \C5 bond wedge & 1.193 & 1.154 & -0.039 & -3.299 & 1.262 & 0.069 & 5.783 \\
\hline $4 \mathrm{H} 8$ bond wedge & 0.948 & 0.979 & 0.031 & 3.319 & 0.914 & -0.034 & -3.586 \\
\hline $\mathrm{C} 5$ - H9 bond bundle & 2.136 & 2.132 & -0.004 & -0.175 & 2.118 & -0.018 & -0.850 \\
\hline \C5 bond wedge & 1.188 & 1.157 & -0.031 & -2.583 & 1.200 & 0.012 & 1.019 \\
\hline$\measuredangle \mathrm{H} 9$ bond wedge & 0.948 & 0.975 & 0.027 & 2.840 & 0.918 & -0.030 & -3.190 \\
\hline Total & 33.772 & 33.718 & -0.054 & -0.159 & 33.652 & -0.120 & -0.354 \\
\hline
\end{tabular}

other. For all three, the region directly above and below the $\mathrm{C}=\mathrm{C}$ bond path intersection with the sphere-corresponding to the $\pi$-bond-appear to be the among the most responsive, making the $\mathrm{C} p$-orbitals seem like sails catching electronic wind. The compliance of the $p$ orbital region is also evident in the high charge density shifts to/from the $\mathrm{C}=\mathrm{C}$ bond wedge regions resulting from the $y$-field in Figure 5. The offsetting \pm signs of $\Delta \mathcal{P}_{x}$ and $\Delta \mathcal{P}_{z}$ about the $\mathrm{C}=\mathrm{C}$ bond path in Figure 6 graphically show why only $\Delta \mathcal{P}_{y}$ achieves a (high) non-zero regional value. Otherwise, the other observations from Figure 5 are also evident: $\Delta \mathcal{P}_{x}$ 
shows a preference for accumulation on the $+x$ (left) side of the sphere; $\Delta \mathcal{P}_{y}$ is primarily positive for $\mathrm{C} 2$, and negative regions are primarily in the $\mathrm{C}-\mathrm{H}$ maximum basins, while the opposite applies to $\mathrm{C} 1$; and $\Delta \mathcal{P}_{Z}$ shows that charge shifted opposite the field direction.

This key finding here is that picturing the response of simple negative charges to an electric field can be a helpful guide in many instances, such as in low-density Van der Waals regions. This is significant because

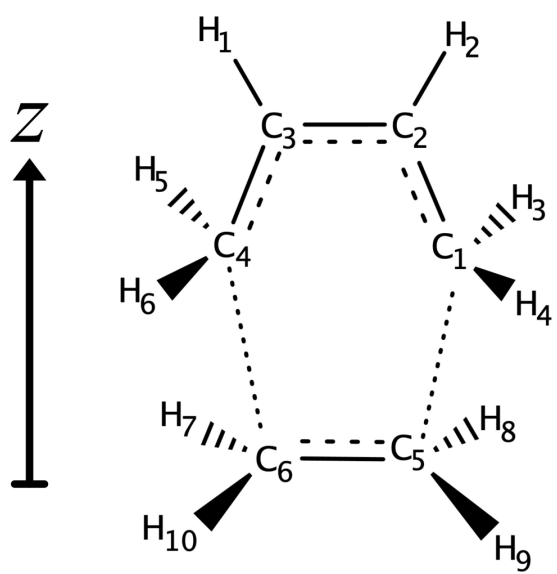

Scheme 4. Numbering and orientation of atoms in TS structure. the response of these regions appears to dictate the response of the inner valance molecular charge redistribution. This interdependent behavior was not evident in the inter-atomic charge transfer, but using the combined approach of atomic and bond regional property analysis, we arrived at a clear picture of the ethylene electric field response.

\section{Diels-Alder electric field catalysis}

Turning now to the charge density response accompanying the catalytic effect of a properly oriented electric field on the cycloaddition of ethylene and cis-butadiene. Recall that

Table 4. Atomic basin and bond bundle condensed valance electron density values for atoms in the R, TS, and $P$ states, i.e. before, during, and after the reaction. $\Delta_{R}$ values indicate the difference from the $R$ state.

\begin{tabular}{|c|c|c|c|c|c|c|c|}
\hline \multirow{3}{*}{ Region } & \multicolumn{7}{|c|}{$\rho_{v}[\mathrm{e}]$} \\
\hline & $\mathrm{R}$ & & TS & & & $P$ & \\
\hline & & & $\Delta_{R}$ & $\% \Delta_{R}$ & & $\Delta_{R}$ & $\% \Delta_{R}$ \\
\hline $\mathrm{C} 1(\mathrm{C} 4)$ atomic basin & 4.038 & 4.074 & 0.036 & 0.887 & 3.983 & -0.055 & -1.354 \\
\hline C2 (C3) atomic basin & 4.014 & 4.036 & 0.022 & 0.544 & 4.047 & 0.033 & 0.818 \\
\hline C5 (C6) atomic basin & 4.032 & 4.050 & 0.018 & 0.441 & 3.974 & -0.057 & -1.425 \\
\hline $\mathrm{H} 1(\mathrm{H} 2)$ atomic basin & 0.945 & 0.953 & 0.008 & 0.838 & 0.960 & 0.015 & 1.542 \\
\hline H3 (H5) atomic basin & 0.937 & 0.944 & 0.007 & 0.720 & 0.968 & 0.031 & 3.299 \\
\hline $\mathrm{H} 4 \mathrm{H6}$ ) atomic basin & 0.948 & 0.954 & 0.005 & 0.541 & 0.970 & 0.022 & 2.273 \\
\hline $\mathrm{H7}(\mathrm{H} 8)$ atomic basin & 0.942 & 0.948 & 0.007 & 0.710 & 0.986 & 0.045 & 4.729 \\
\hline $\mathrm{H} 10(\mathrm{H} 9)$ atomic basin & 0.942 & 0.948 & 0.007 & 0.711 & 0.980 & 0.039 & 4.093 \\
\hline Total & 33.596 & 33.814 & 0.217 & 0.647 & 33.738 & 0.142 & 0.421 \\
\hline$\overline{\mathrm{C} 1-\mathrm{C} 2 \text { bond bundle }}$ & 3.258 & 2.981 & 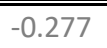 & -8.502 & 2.161 & $\begin{array}{c}-1.097 \\
\end{array}$ & 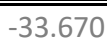 \\
\hline $\mathrm{C} 1-\mathrm{C} 5$ bond bundle & 0.000 & 0.312 & 0.312 & --- & 1.971 & 1.971 & -- \\
\hline $\mathrm{C} 1-\mathrm{H} 3$ bond bundle & 2.118 & 2.161 & 0.043 & 2.054 & 2.054 & -0.064 & -3.008 \\
\hline $\mathrm{C} 1-\mathrm{H} 4$ bond bundle & 2.170 & 2.140 & -0.030 & -1.397 & 1.954 & -0.216 & -9.951 \\
\hline $\mathrm{C} 2-\mathrm{C} 3$ bond bundle & 2.387 & 2.785 & 0.398 & 16.665 & 3.401 & 1.014 & 42.480 \\
\hline $\mathrm{C} 2-\mathrm{H} 2$ bond bundle & 2.144 & 2.137 & -0.007 & -0.328 & 2.132 & -0.012 & -0.558 \\
\hline $\mathrm{C} 5-\mathrm{C} 6$ bond bundle & 3.306 & 2.970 & -0.336 & -10.175 & 1.973 & -1.333 & -40.332 \\
\hline $\mathrm{C} 5-\mathrm{H} 8$ bond bundle & 2.131 & 2.142 & 0.011 & 0.500 & 2.011 & -0.120 & -5.614 \\
\hline $\mathrm{C} 5-\mathrm{H} 9$ bond bundle & 2.131 & 2.136 & 0.005 & 0.250 & 1.983 & -0.147 & -6.921 \\
\hline Total & 33.596 & 33.772 & 0.176 & 0.524 & 33.907 & 0.311 & 0.926 \\
\hline
\end{tabular}




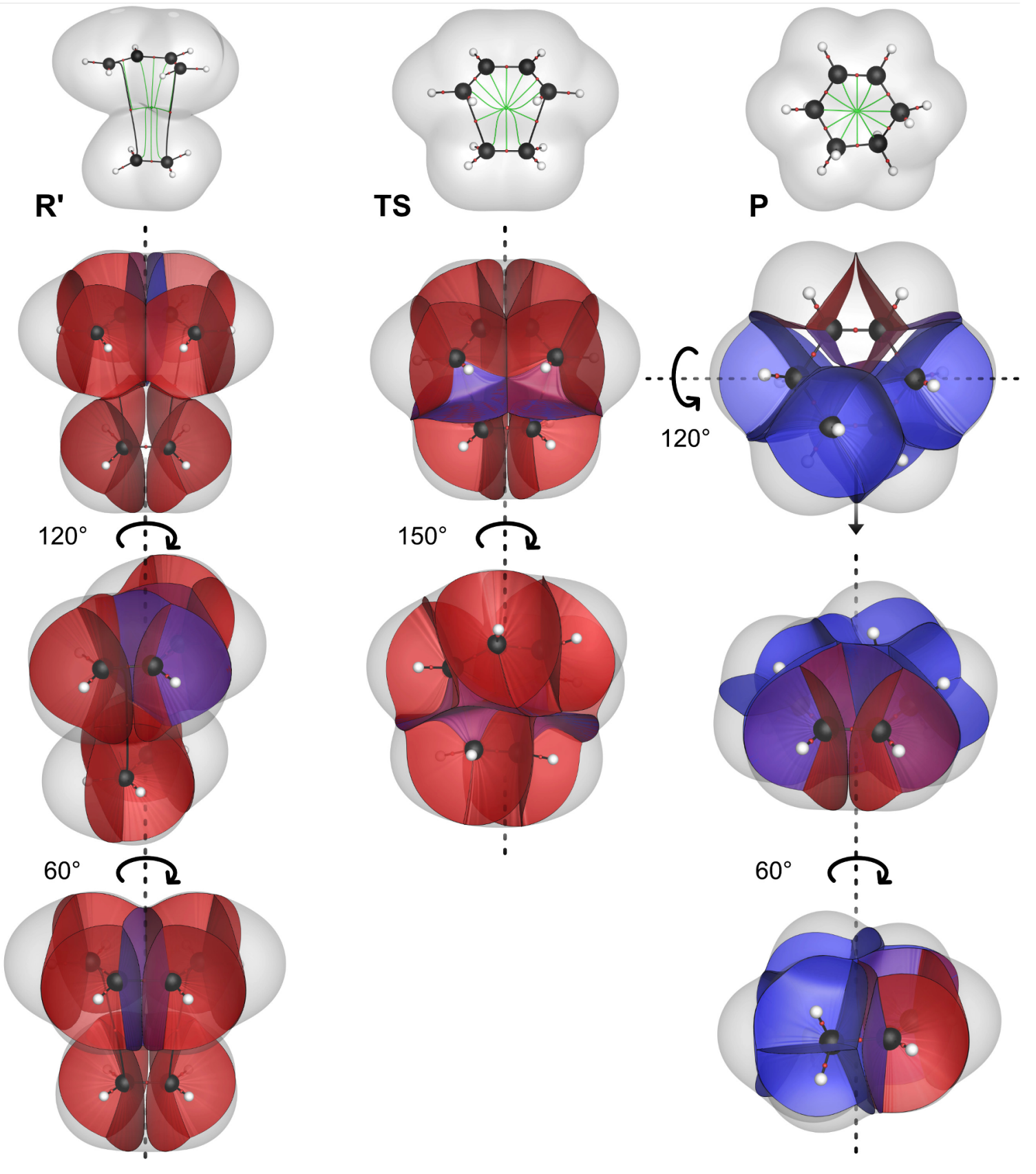

Figure 7. Bond bundle surfaces for the reactant complex (R'), transition state (TS), and product (P) optimized geometries. In the $\mathrm{R}$ column, the $\mathrm{C}-\mathrm{C}$ single bond surfaces are shaded blue. In the TS column, the surfaces of the bonds formed in the reaction are emphasized and shaded blue/purple. In the $P$ column, the $C=C$ double bond surfaces are shaded red.

the effect itself was observed as a lowering of the reaction barrier-i.e. of the TS energy-so we will analyze the atomic and bond bundle charge density response of the TS structure shown in Scheme 4. Meir et al. found that the electric field pointing in the negative $z$ direction, from the butadiene to the ethylene, lowered the barrier while the opposite effect was not observed for the opposite field [6]. Our calculations gave results mostly in agreement, but both fields were found to stabilize the TS relative to the reactant sum (R; also with the same field applied). The NEF barrier was lowered $5.9 \mathrm{kcal} / \mathrm{mol}$ by the $-z$ field and $2.2 \mathrm{kcal} / \mathrm{mol}$ by the $+z$ field, so 
the $-z$ field has a stronger stabilizing effect, agreeing with the referenced investigation.

Table 3 lists the atomic basin and bond bundle condensed valence electron density $\left(\rho_{v}\right)$ values for the TS structure with/without the $\pm z$ fields. The total valence density of an atom is equal to its total density minus its core density, $\rho_{v}=\rho-\rho_{\text {core }}$. The gradient bundle condensed valence density is then calculated, similar to the condensed deformation energy of Figure 4, by subtracting off the spherical atomic reference amount of core density from each gradient bundle, $\mathcal{P}_{v}=\mathcal{P}-\mathcal{P}_{\text {core,sph }}$. Note that symmetry degenerate atomic basins have been averaged, as indicated parenthetically in the Region column.

Starting with atomic basins, we see a response similar to that of the total charge density in ethylene. Inter-atomic valance charge transfer is never more than a little under a twentieth of an electron, and the prevalent motion is opposite the field. Overall, charge shifts from the butadiene central $\mathrm{C}_{2,3}$ and $\mathrm{H}_{1,2}$ atoms to the ethylene $\mathrm{C}$ and $\mathrm{H}$ atoms, and the $\Delta$ values for every atom are nearly equal and opposite with respect to field direction. From a pairwise perspective, there is also a lesser transfer between the ethylene $\mathrm{C}$ atoms and the butadiene $\mathrm{C}_{1,4}$ atoms. At first glance, this appears to be another case where the electron gas behavior would serve as a good predictor and explanation of the response. Regarding the charge density behavior responsible for the shift in the reaction barrier, however, it is difficult to say whether the apparent inter-atomic $\mathrm{C} 1-\mathrm{C} 5$ charge transfer should have an effect on the barrier, or in what direction that effect should manifest.

When bond bundle properties are considered, we can interpret the regional electric field responses in the context of valence bond theory. As an example and benchmark for the electric field response, Table 4 lists regional valence electron counts for the NEF system in the reactant, transition, and product states, and Figure 7 depicts the corresponding bond bundle surfaces. As shown in Scheme 2, the forward reaction direction is that which decreases the ethylene and butadiene $\mathrm{C}=\mathrm{C}$ bonds from double to single bond order, while increasing that of the butadiene C2-C3 from single to double. At the TS (Scheme 4), the ethylene and butadiene $\mathrm{C}-\mathrm{C}$ bonds have an intermediate bond order between 1 and 2, while the inter-molecular $\mathrm{C} 1-\mathrm{C} 5$ and C4-C6 bonds have a bond order between 0 and 1. Recalling that $\mathrm{C}-\mathrm{C}$ bond order corresponds to bond bundle valance electron count [11], here the bond bundle valence density throughout the reaction recovers our preconceived notions. The $\mathrm{C}=\mathrm{C}$ bonds decrease by 1.1 and 1.3 valance electrons in butadiene and ethylene respectively ( $34 \%$ and $40 \%)$, while the C2C3 bond increases by 1 electron (42\%) - a clear shift in "order" in expected direction and magnitude. 
Although intermolecular $\mathrm{C} \cdots \mathrm{C}$ bond paths are present in the $\mathrm{R}$ state, from a bond bundle perspective the bonds explicitly form before the TS (they don't exist in the R state) and populate to the expected $\sim 2$ valance electrons of a $\mathrm{C}-\mathrm{C}$ single bond in the product. Inspection of the intermolecular bond bundle surfaces in the R state (Figure 7) reveals that the ethylene bond bundle surfaces look identical to those in the lone molecule (Figure 2), and that the intermolecular $\mathrm{C} \cdots \mathrm{C}$ bond paths appear to instead coincide with the ethylene $\mathrm{C}=\mathrm{C}$ bond bundle surfaces. We have previously shown that bond paths need not map to maximum basins in $\mathcal{P}$ and hence need not correspond to bond bundles $[2,4]$. This allows one to assess bond bundle properties with the knowledge that the reference state for a bond is to not exist.

Considering the bond bundle valence electron counts at the TS, using Hammond's Postulate we would anticipate that the TS of an exothermic reaction more closely resemble the reactant state, to which it is nearer in energy, than the product state. Here, Table 4 confirms this intuition, indicating that the TS is roughly one third of the way along the reaction coordinate. This is based on the $\Delta_{\mathrm{R}}$ values of the transitioning C-C bonds mentioned above, which have changed at the TS by about a third of their respective changes in the P state- - for example, the C5-C6 bond loses 1.33 electrons in the reaction, but has only lost 0.34 at the TSexcept the intermolecular C-C bonds which have changed by about a sixth of their eventual quantities. This is more than some abstract notion of the location of the TS along the reaction coordinate. Initial and final bond states, together with the redistribution of charge density that accompanies a chemical reaction, is the chemical reaction. When bonds are represented numerically, it is typically in thermodynamic terms, but it is understood that electronic motion between potential states is the thing being described. Using bond bundle analysis, the conceptual bond representation gains rigorous real-space boundaries and numerous quantifiable properties including explicit quantities of charge and energy that are redistributed within and between bonds.

Now that we have an idea of the basic bond properties in the reaction and how they map to the conventional chemical depiction of the accompanying bond order transitions, we can contextualize the electric field response of the bonds in the TS. Table 3 shows that in response to the $-z$ field, the butadiene $\mathrm{C} 1-\mathrm{C} 2$ and ethylene $\mathrm{C} 5-\mathrm{C} 6$ bond bundle valance electron counts decrease by 0.044 and 0.105 electrons respectively, while the $\mathrm{C} 2-\mathrm{C} 3$ value increases by 0.032 valance electrons. Regarding the significance of these values, note that they represent a shift in valence charge for these bonds equal to between roughly 10 to $30 \%$ of their respective $\Delta_{R}$ TS values in Table 4. For example, the C5-C6 bond loses 0.105 valance electrons due to the 
-z field, in addition to the 0.34 valance electrons it has already lost relative to the reactant state. The redistribution resulting from the $+z$ field (which Meir et al. found to not raise or lower the reaction barrier [6]) also includes a lowered butadiene C1-C2 valance electron count, but in this case the $\mathrm{C} 2-\mathrm{C} 3$ and $\mathrm{C} 5-\mathrm{C} 6$ counts shift opposite the direction of that implied by the forward reaction and observed in the bond bundle values in Table 4. So, the more strongly catalyzing field for the forward reaction is that which shifts $\mathrm{C}-\mathrm{C}$ bond orders in the TS to look more like they will in the product state, while the effect of the $+z$ field is accompanied by bond order changes that are contradictory in this regard. This is again consistent with Hammond's Postulate, and we see that the catalyzing field simply "pushes" the TS density in the direction of the product state. Table 4 indicates that, for the NEF system, once the bonds in the system reach that specific TS level of valance electron population $\left(\rho_{v, \mathrm{TS}}\right)$, the TS has been reached. The remaining (majority) change in bond bundle valance density necessary to achieve the product state thus occurs on the "downhill" side of the reaction. The $-z$ field, by pushing and pulling charge in the reaction direction, is causing $\rho_{v, \mathrm{TS}}$ to be achieved—and thus making the downhill side of the reaction accessible — earlier along the reaction coordinate.

The newly formed C1-C5 and C4-C6 bonds experience a shift counter to the reaction direction, decreasing instead of increasing; a countereffect of the shifts between $\mathrm{C}-\mathrm{C}$ single and double bond order states which constitute the primary electric field response. This concurs with the observation from Figure 6 that the most mobile regions of $\mathcal{P}$ - for both accumulation and depletion - are those corresponding to the $p$-orbital $\pi$-bonding regions of $\mathrm{C}=\mathrm{C}$ bonds.

Otherwise, we again see in Table 3 that the $\mathrm{C}$ intra-atomic charge redistribution can be counter that expected from a simple Coulombic approach. In response to the $-z$ field, the $\mathrm{C} 1$ atom shifts charge primarily from its $\mathrm{C} 1-\mathrm{C} 2$ to its $\mathrm{C} 1-\mathrm{C} 5$ bond wedges, opposite the direction of the system as a whole. Its response to the $+z$ field, however, is not to shift charge in the opposite direction, but to instead shift charge from both C1-C2 and C1-C5 bond wedges into its $\mathrm{C}-\mathrm{H}$ bond wedges. The response of the ethylene $\mathrm{C} 5$ atom, however, is also opposite the expected $-z$ field response, shifting charge from its $\mathrm{C}$ bonds to its $\mathrm{H}$ bonds, but in this case its response is equal and opposite with respect to field direction. On this basis we again conclude that $\mathrm{C}$ atom intra-atomic charge redistribution is secondary to the primary low-lying density electric field response, which here too is predicted by an electron gas approximation. Note that, in this case, the newly formed C1-C5 bond, when analyzed as a standalone volume, has charge shifting in the expected electron gas direction, opposite that of the applied field, so this low-order bond region (with only $\sim 0.3$ valance electrons) responds as do other low-lying 
density regions.

As in the analysis of the ethylene electric field response, the primary effect is the intraatomic redistribution of $\mathrm{C}$ electron density. In the transition state context, changes in bond bundle condensed valence density provided a straightforward chemical interpretation as to why this charge density response should catalyze the reaction: The catalyzing $-z$ field gives rise to charge redistribution between $\mathrm{C}$ atom bond wedges such that the TS density more closely resembles that of the product, effectively using Hammond's Postulate to shorten the charge redistribution "distance" between the reactant and product states.

\section{Conclusions}

The bridge between QTAIM and other branches of experimental and theoretical chemistry and materials science rests largely on its ability to produce atomic (and therefore molecular and crystalline) regional properties that can be readily compared to other results [1,3]. Welldefined regional energies are a quality of any region bounded by zero flux surfaces, and such regions can be generated arbitrarily. Taking this property to the limit, the gradient bundle decomposition method is the differential partitioning of $\rho$ into infinitesimal zero-flux surface bounded regions, producing the gradient bundle condensed energy, charge density and other condensed properties. The topology of the condensed density also uniquely defines the surfaces of charge density bonding regions called bond bundles.

Here we showed that the bond bundle decomposition method allows for the direct qualitative and quantitative inspection of the distribution and redistribution of charge density that accompany static and dynamic chemical bonding. Applied to the toy problem of electric field catalysis, and the more general problem of the charge density response to an external electric field, we observed that regional property shifts between bond wedges and bond bundles are more dynamic in both sign and magnitude than are atomic basin regional shifts, and that they allow for immediate chemical interpretation. In the analysis of both the ethylene and TS electric field response, we saw that $\mathrm{C}$ atom intra-atomic redistribution can be counter that of the overall molecular response, and that the most compliant regions of condensed charge density are those that correspond to the $\pi$-bonding component of $\mathrm{C}=\mathrm{C}$ double bonds. In the analysis of the TS field response, we saw that the catalyzing field achieves its catalytic effect by preferentially accumulating and depleting charge in the same direction as the reaction profile itself, thus allowing the TS valance density bond configuration to be achieved at a lower energy 
cost.

These results are promising, but anecdotal. The data generated using this methoddemonstrated here to be useful for extracting meaningful chemical interpretations from a small number of electron charge density grids - can be generated in ever-growing quantities. Moving forward, we plan on incorporating methods like principal component analysis and machine learning-based regression in order to uncover charge density structure-property relationships either too subtle or occurring at too large a scale (i.e. emergent properties) to be detected through direct inspection, with in-progress investigations looking again into the charge density electric field response in a biological context. The biochemical community is making great strides in understanding the significant role of electric fields in enzymatic catalysis [23], which involves countless chemical scenarios not unlike that treated in this work. We hope to aid in the process of discovery with the dual ability of local direct inspection within and large-scale correlation across enzyme active sites.

\section{Acknowledgements}

This work was supported by the Office of Naval Research grant N00014-10-1-0838.

\section{References}

[1] R. F. W. Bader, Atoms in Molecules: A Quantum Theory (Clarendon Press, Oxford, 1990).

[2] T. R. Wilson, M. Rajivmoorthy, J. Goss, S. Riddle, and M. E. Eberhart, Observing the 3D Chemical Bond and Its Energy Distribution in a Projected Space, ChemPhysChem 20, 3289 (2019).

[3] C. F. Matta and R. J. Boyd, editors, The Quantum Theory of Atoms in Molecules: From Solid State to DNA and Drug Design (Wiley-VCH Verlag GmbH \& Co. KGaA: Weinheim, 2007).

[4] T. R. Wilson and M. E. Eberhart, Quantum Theory of Atoms in Molecules in Condensed Charge Density Space, Can. J. Chem. 97, 757 (2019).

[5] T. Wilson, A. Alexandrova, and M. Eberhart, Electron Density Geometry and the Quantum Theory of Atoms in Molecules, (2021).

[6] R. Meir, H. Chen, W. Lai, and S. Shaik, Oriented Electric Fields Accelerate Diels-Alder Reactions and Control the Endo/Exo Selectivity, ChemPhysChem 11, 301 (2010).

[7] S. Yu, P. Vermeeren, T. A. Hamlin, and F. M. Bickelhaupt, How Oriented External Electric Fields Modulate Reactivity, Chem. - Eur. J. 27, 5683 (2021).

[8] N. G. Léonard, R. Dhaoui, T. Chantarojsiri, and J. Y. Yang, Electric Fields in Catalysis: 
From Enzymes to Molecular Catalysts, ACS Catal. 11, 10923 (2021).

[9] S. Shaik, D. Mandal, and R. Ramanan, Oriented Electric Fields as Future Smart Reagents in Chemistry, Nat. Chem. 8, 1091 (2016).

[10] S. D. Fried, S. Bagchi, and S. G. Boxer, Extreme Electric Fields Power Catalysis in the Active Site of Ketosteroid Isomerase, Science (2014).

[11] T. E. Jones and M. E. Eberhart, The Bond Bundle in Open Systems, Int. J. Quantum Chem. 110, 1500 (2010).

[12] T. E. Jones, M. E. Eberhart, S. Imlay, C. Mackey, and G. B. Olson, Better Alloys with Quantum Design, Phys. Rev. Lett. 109, 125506-(2012).

[13] T. E. Jones, M. E. Eberhart, S. Imlay, and C. Mackey, Bond Bundles and the Origins of Functionality, J. Phys. Chem. A 115, 12582 (2011).

[14] W. H. E. Schwarz, K. Ruedenberg, and L. Mensching, Chemical Deformation Densities. 1. Principles and Formulation of Quantitative Determination, J. Am. Chem. Soc. 111, 6926 (1989).

[15] A. Morgenstern, T. Wilson, J. Miorelli, T. Jones, and M. E. Eberhart, In Search of an Intrinsic Chemical Bond, Comput. Theor. Chem. 1053, 31 (2015).

[16] J. C. Slater, The Virial and Molecular Structure, J. Chem. Phys. 1, 687 (1933).

[17] K. Ruedenberg and M. W. Schmidt, Physical Understanding through Variational Reasoning: Electron Sharing and Covalent Bonding, J. Phys. Chem. A 113, 1954 (2009).

[18] H. M. Aitken and M. L. Coote, Can Electrostatic Catalysis of Diels-Alder Reactions Be Harnessed with PH-Switchable Charged Functional Groups?, Phys. Chem. Chem. Phys. 20, 10671 (2018).

[19] M. Zhang, F. Zhang, H. Xu, and Z. Su, The Regulation of Hydroboration of Olefins by Oriented External Electric Field, New J. Chem. 42, 18402 (2018).

[20] Y. K. Lei, J. Zhang, Z. Zhang, and Y. Q. Gao, Dynamic Electric Field Complicates Chemical Reactions in Solutions, J. Phys. Chem. Lett. 10, 2991 (2019).

[21] C. Acosta-Silva, J. Bertran, V. Branchadell, and A. Oliva, Kemp Elimination Reaction Catalyzed by Electric Fields, ChemPhysChem 21, 295 (2020).

[22] S. D. Fried and S. G. Boxer, Electric Fields and Enzyme Catalysis, Annu. Rev. Biochem. 86, 387 (2017).

[23] M. R. Hennefarth and A. N. Alexandrova, Advances in Optimizing Enzyme Electrostatic Preorganization, Curr. Opin. Struct. Biol. 72, 1 (2022).

[24] A. Morgenstern, M. Jaszai, M. E. Eberhart, and A. N. Alexandrova, Quantified Electrostatic Preorganization in Enzymes Using the Geometry of the Electron Charge Density, Chem. Sci. 8, 5010 (2017).

[25] J. Lameira, R. P. Bora, Z. T. Chu, and A. Warshel, Methyltransferases Do Not Work by Compression, Cratic, or Desolvation Effects, but by Electrostatic Preorganization, Proteins Struct. Funct. Bioinforma. 83, 318 (2015).

[26] S. C. L. Kamerlin, P. K. Sharma, Z. T. Chu, and A. Warshel, Ketosteroid Isomerase Provides Further Support for the Idea That Enzymes Work by Electrostatic Preorganization, Proc. Natl. Acad. Sci. 107, 4075 (2010).

[27] A. C. Aragonès, N. L. Haworth, N. Darwish, S. Ciampi, N. J. Bloomfield, G. G. Wallace, 
I. Diez-Perez, and M. L. Coote, Electrostatic Catalysis of a Diels-Alder Reaction, Nature 531, 88 (2016).

[28] M. T. Blyth and M. L. Coote, A PH-Switchable Electrostatic Catalyst for the Diels-Alder Reaction: Progress toward Synthetically Viable Electrostatic Catalysis, J. Org. Chem. 84, 1517 (2019).

[29] J. B. Siegel et al., Computational Design of an Enzyme Catalyst for a Stereoselective Bimolecular Diels-Alder Reaction, Science (2010).

[30] G. te Velde, F. M. Bickelhaupt, E. J. Baerends, C. Fonseca Guerra, S. J. A. van Gisbergen, J. G. Snijders, and T. Ziegler, Chemistry with ADF, J. Comput. Chem. 22, 931 (2001).

[31] C. Fonseca Guerra, J. G. Snijders, G. te Velde, and E. J. Baerends, Towards an Order-N DFT Method, Theor. Chem. Acc. 99, 391 (1998).

[32] E. J. Baerends et al., ADF (Theoretical Chemistry, Vrije Universiteit, Amsterdam, The Netherlands, 2016).

[33] J. P. Perdew, K. Burke, and M. Ernzerhof, Generalized Gradient Approximation Made Simple, Phys. Rev. Lett. 77, 3865 (1996).

[34] A. Klamt and G. Schüürmann, COSMO: A New Approach to Dielectric Screening in Solvents with Explicit Expressions for the Screening Energy and Its Gradient, J. Chem. Soc. Perkin Trans. 2799 (1993).

[35] A. Klamt, Conductor-like Screening Model for Real Solvents: A New Approach to the Quantitative Calculation of Solvation Phenomena, J. Phys. Chem. 99, 2224 (1995).

[36] A. Klamt and V. Jonas, Treatment of the Outlying Charge in Continuum Solvation Models, J. Chem. Phys. 105, 9972 (1996).

[37] N. L. Allinger, X. Zhou, and J. Bergsma, Molecular Mechanics Parameters, J. Mol. Struct. THEOCHEM 312, 69 (1994).

[38] Tecplot Inc., Tecplot $3602013 R 1$ (Tecplot Inc., 2013), https://www.tecplot.com.

[39] T. R. Wilson and M. E. Eberhart, Bondalyzer (First Principles Materials Design and Software, Golden, $\mathrm{CO}$, 2021), https://github.com/moltheorygroup/BondalyzerTecplotAddon. 